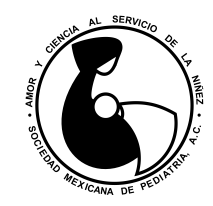

\title{
¿Sepsis neonatal con hiperamonemia? Diagnóstico clínico precoz de una acidemia isovalérica neonatal
}

\author{
Óscar Sánchez, ${ }^{1}$ Lissete Cabarcas, ${ }^{2}$ Eugenia Espinosa, ${ }^{2}$ \\ Johana María Guevara, ${ }^{3}$ Olga Yaneth Echeverri-Peña ${ }^{3, *}$ \\ ${ }^{1}$ Universidad Militar Nueva Granada; ${ }^{2}$ Servicio de Neurología Pediátrica. Instituto Roosevelt; ${ }^{3}$ Instituto de Errores Innatos \\ del Metabolismo. Pontificia Universidad Javeriana. Bogotá, Colombia.
}

\begin{abstract}
RESUMEN
Introducción: La aciduria isovalérica (IVA) es un error innato del metabolismo dentro del grupo de acidemias orgánicas (AO). Es secundaria a la deficiencia de la isovaleril-CoA-deshidrogenasa, y tiene una prevalencia de aproximadamente un caso por cada 100,000 nacidos vivos. La IVA de inicio neonatal es más grave que cuando la presentación es más tardía. Presentación del caso: Se trata de un recién nacido masculino en quien se realizó el diagnóstico de IVA, dado el cuadro clínico de afección neurológica, acidosis metabólica e hiperamonemia dentro de la primera semana de vida. Se inició manejo dietético especializado y el paciente evolucionó satisfactoriamente. Conclusiones: El diagnóstico oportuno de IVA puede prevenir secuelas y desenlaces fatales cuando se ofrece tratamiento temprano.
\end{abstract}

Palabras clave: Acidemia isovalérica, acidemia orgánica, hiperamonemia neonatal, error innato del metabolismo, acidosis metabólica.

\section{INTRODUCCIÓN}

Las acidemias orgánicas (AO) son errores innatos del metabolismo causados por deficiencias en proteínas involucradas en las vías metabólicas intermediarias de los carbohidratos, aminoácidos y ácidos grasos que producen acumulación de ácidos orgánicos en diferentes tejidos y su excreción elevada en orina. ${ }^{1}$ Aunque la prevalencia e incidencia de las $\mathrm{AO}$ son bajas, ${ }^{2}$ hay

\begin{abstract}
Introduction: Isovaleric aciduria $(\mathrm{IA})$ is an inborn error of metabolism within the group of organic acidemias (AO). It is secondary to isovaleryl-CoA dehydrogenase deficiency, and has a prevalence that is around one case per 100,000 live births. Neonatal onset IA is more severe than when the presentation is at older ages. Case presentation: We present the case of a male newborn in whom the diagnosis of IA was made, given the clinical picture of neurological condition, metabolic acidosis and hyperammonemia within the first week of life. Specialized dietary management was initiated and the patient evolved satisfactorily. Conclusions: Timely diagnosis of IA can prevent sequelae and fatal outcomes, when early treatment is offered.
\end{abstract}

Keywords: Isovaleric acidemia, organic acidemia, neonatal hyperammonemia, innate error of metabolism, metabolic acidosis.

regiones del mundo con mayor frecuencia de presentación, como en India y otros países del Oriente Medio. En Inglaterra se estima una incidencia de 3.7 casos por cada 100,000 recién nacidos vivos. ${ }^{1}$

Con la implementación en varios países del tamizaje metabólico neonatal se ha observado un aumento de los casos detectados, ${ }^{3}$ lo cual sugiere que las AO se han subdiagnosticado, particularmente en países donde no se realiza esta prueba. Cabe resaltar que sin un diag-

\footnotetext{
* Correspondencia: OYEP, oyecheve@javeriana.edu.co

Conflicto de intereses: Los autores declaran que no tienen.

Citar como: Sánchez O, Cabarcas L, Espinosa E, Guevara JM, Echeverri-Peña OY. ¿Sepsis neonatal con hiperamonemia? Diagnóstico clínico precoz de una acidemia isovalérica neonatal. Rev Mex Pediatr. 2019; 86(5):197-201.

[Neonatal sepsis with hyperammonemia? Early clinical diagnosis of a neonatal isovaleric acidemia]
} 
nóstico y manejo oportuno en las primeras 24 a 48 horas que inician los síntomas, el pronóstico se ensombrece con mayor probabilidad de complicaciones neurológicas a largo plazo, progresión a coma y muerte. ${ }^{4,5}$

La acidemia isovalérica (IVA) es una de las AO clásicas. Se presenta como consecuencia de la deficiencia genética de la isovaleril-CoA-deshidrogenasa, la cual es responsable de catalizar el tercer paso del metabolismo de la leucina. El bloqueo de la vía genera la acumulación de metabolitos tóxicos derivados del isovaleril-CoA como el ácido valérico, el ácido 3-hidroxi-isovalérico, la isovalerilcarnitina y la isovalerilglicina (Figura 1). ${ }^{6}$

El presente reporte corresponde a un paciente en quien se realizó el diagnóstico de IVA en las primeras 48 horas en que se presentaron los síntomas.

\section{PRESENTACIÓN DEL CASO}

Recién nacido masculino, producto de la primera gestación de padres jóvenes consanguíneos (segundo grado) sin otros antecedentes perinatales de relevancia. Durante los primeros cinco días de vida el paciente se encontró sin problemas; al sexto día, mostró irritabilidad, somnolencia y succión débil.

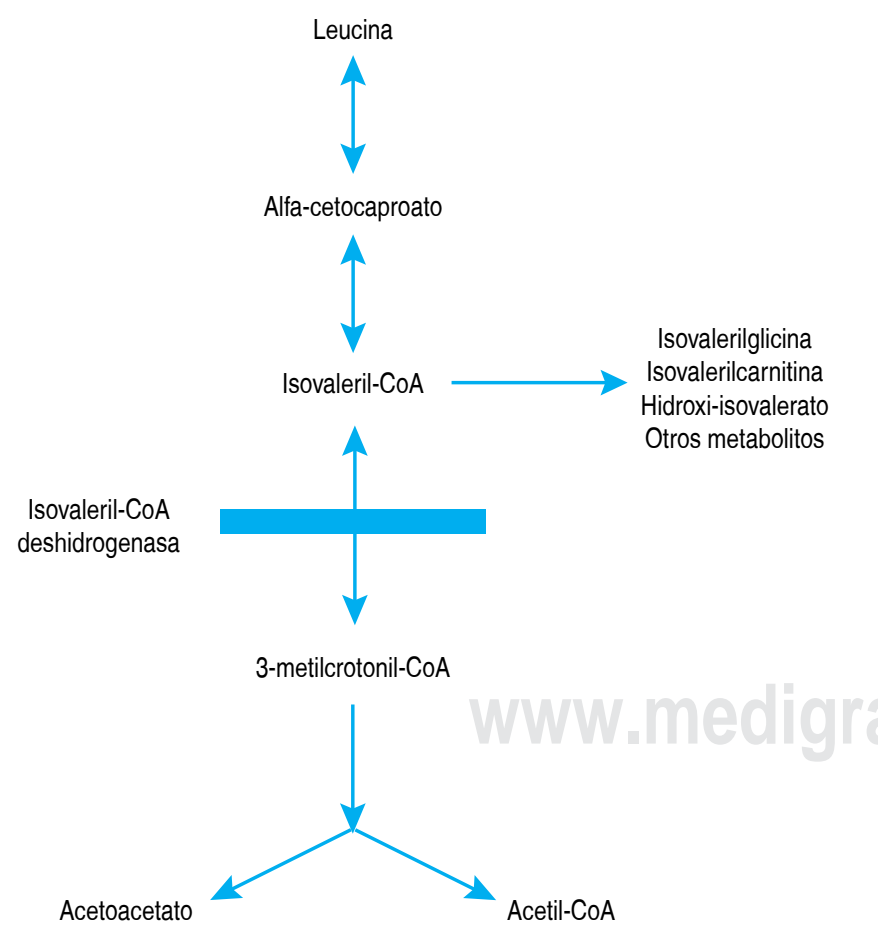

Figura 1: Vía metabólica de la leucina. Con una barra se indica el sitio del bloqueo metabólico en IVA.
Fue llevado para atención médica y se hospitaliza en la Unidad de Cuidados Neonatales por sospecha de sepsis neonatal. Al examen físico, el peso, talla y perímetro cefálico eran adecuados para la edad; sin embargo, se observó una pérdida de peso mayor de lo esperado en la primera semana de vida, signos vitales normales, sin alteraciones en el patrón respiratorio, cardiaco o síntomas gastrointestinales. No obstante, estaba somnoliento e hipoactivo con reflejos primitivos disminuidos e incompletos. En los exámenes de laboratorio se evidenció hiperglucemia $(182 \mathrm{mg} / \mathrm{dL})$ y acidosis metabólica con anión GAP elevado (no se dispone de datos numéricos), ante estos hallazgos se inició manejo antibiótico con gentamicina y ampicilina, soporte con líquidos endovenosos y reposición de bicarbonato.

La respuesta clínica no fue satisfactoria; continuaba somnoliento e hipoactivo. En la determinación de amonio se observó un valor de $292 \mu \mathrm{mol} / \mathrm{L}$, (límite superior: $100 \mu \mathrm{mol} / \mathrm{L}$ ), por lo que se da manejo para hiperamonemia: se suspende la vía oral con ajuste del flujo metabólico y se indica ácido carbaglúmico. Se realizaron tres controles de amonio en las siguientes 24 horas, con valores de $292 \mu \mathrm{mol} / \mathrm{L}, 31.86 \mu \mathrm{mol} / \mathrm{L}$ y 31.27 $\mu \mathrm{mol} / \mathrm{L}$, respectivamente. Se completaron los estudios con aminoácidos cuantitativos en plasma por HPLC, cromatografía de ácidos orgánicos en orina, lactato y piruvato séricos.

Los aminoácidos cuantitativos en plasma, lactato y piruvato se reportaron dentro de límites normales, en la cromatografía de ácidos orgánicos en orina se observó elevada excreción de isovalerilglicina, ácidos 3-hidroxiisovalérico, isovalerilglutámico y 2 -hidroxi-isovalérico. Con este perfil metabólico se diagnosticó acidemia isovalérica (Figura 2), por lo que se indica fórmula especializada libre de leucina, dieta con restricción proteica y L-carnitina, como coadyuvante. Así, el paciente mostró respuesta clínica y bioquímica favorable, con amonio dentro de valores normales y corrección de la acidosis metabólica. Se egresó alerta y activo.

A los seis meses de edad, al tratamiento se adicionó L-glicina y L-leucina. Esta última la recibió por un periodo corto de tiempo, pues no se identificó una clara indicación. Se solicitó cromatografía de aminoácidos cualitativos en plasma, los cuales fueron normales. El perímetro cefálico, peso y talla se encontraban entre - 2 y -3 desviaciones estándar, pero persistía con hipotonía. Sin embargo, el neurodesarrollo era acorde a la edad, logrando sedestación en trípode, prensión palmar radial de objetos de forma simétrica, balbuceo y búsqueda de interacción con sonrisas. 


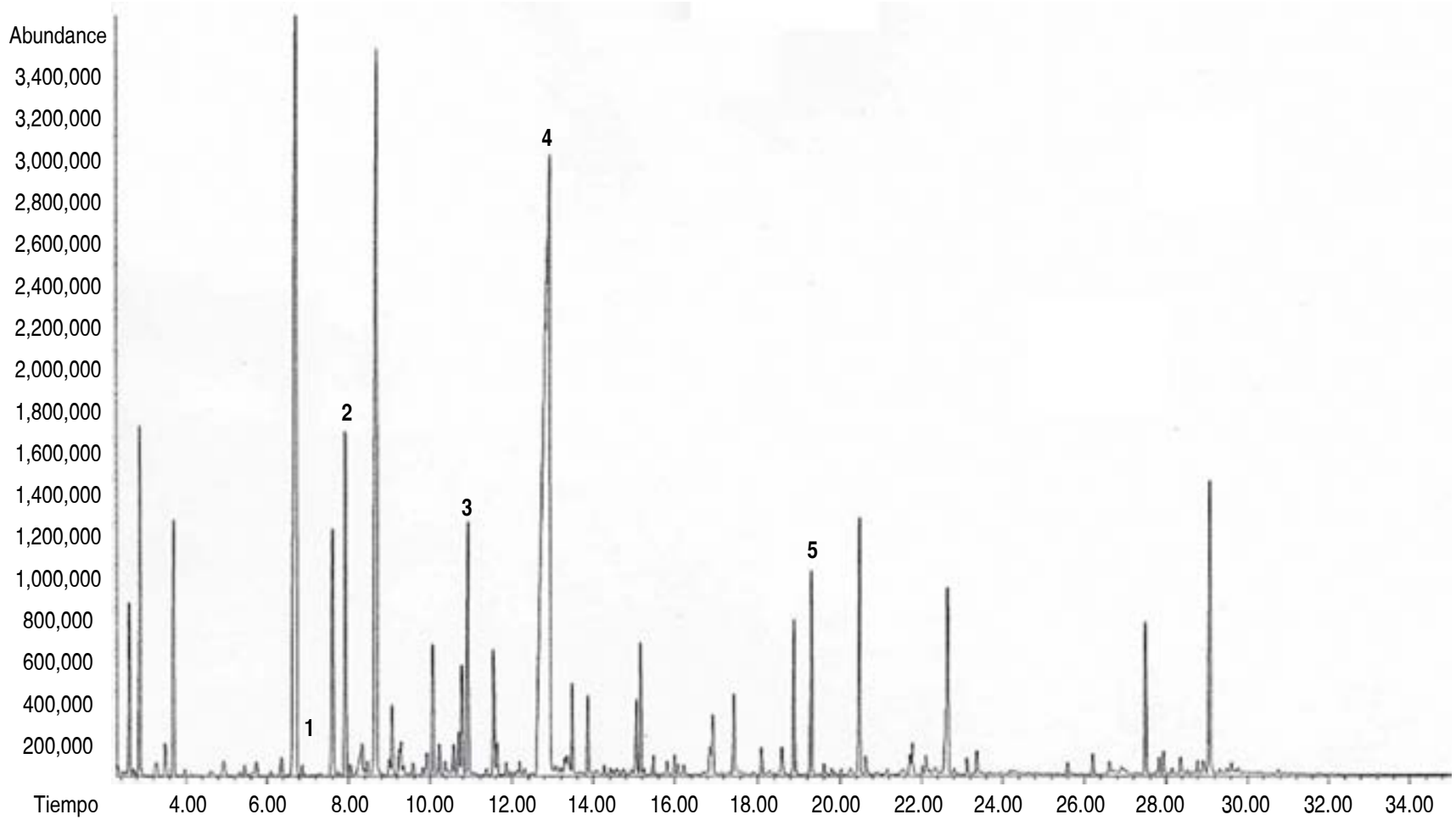

Figura 2: Cromatograma correspondiente al perfil de ácidos orgánicos urinarios del paciente.

\section{DISCUSIÓN}

La mitad de los pacientes con IVA inicia la sintomatología dentro las primeras cinco semanas de edad, la cual se caracteriza por succión débil, vómito, acidosis metabólica, cetosis, deterioro progresivo del estado de conciencia, crisis convulsivas y, de continuar, puede progresar coma y muerte. La otra mitad de los pacientes inicia los síntomas cuando son lactantes o preescolares y algunos pocos en la adolescencia. En otros países se han reportado pacientes asintomáticos detectados mediante el tamizaje metabólico neonatal ${ }^{7}$ en Colombia este tipo de tamizaje no está disponible, por lo que sólo se detectan pacientes con manifestaciones clínicas establecidas.

Varios pacientes con algún tipo de AO, como ocurrió en el caso descrito, son diagnosticados con sepsis neonatal temprana, esto se debe principalmente a la poca especificidad de los síntomas y signos en el periodo neonatal; por lo tanto, es importante tener la sospecha clínica para el diagnóstico, prestando especial atención a los pacientes que no responden de manera adecuada al manejo convencional o en quienes predomina el compromiso neurológico. ${ }^{8}$
Al igual que en otros errores innatos del metabolismo, la IVA presenta un espectro metabólico y clínico amplio, de tal manera que el compromiso metabólico da lugar a la división en dos grandes grupos, de acuerdo a los niveles de $\mathrm{N}$-isovalerilcarnitina en el tamizaje neonatal: IVA con compromiso metabólico grave y otro grupo con compromiso leve. ${ }^{9}$

El espectro de las manifestaciones puede ser de leve a grave; los pacientes con un cuadro leve tienen poca afección neurológica o en el neurodesarrollo. Para quienes tienen un cuadro clínico moderado, el compromiso neurológico es mayor y con algún grado de retraso en el neurodesarrollo, el cual puede ser reversible con el tiempo. Los pacientes con mayor gravedad tienen las manifestaciones neurológicas más importantes y con marcado retraso en el neurodesarrollo. ${ }^{10} \mathrm{El}$ compromiso metabólico en nuestro paciente, a pesar de no disponer de los niveles de $\mathrm{N}$-isovalerilcarnitina, probablemente fue severo. La cromatografía de ácidos orgánicos arrojó un perfil clásico de IVA asociado a manifestación clínica grave, dado el compromiso neurológico y la instauración temprana de los síntomas.

Como diagnósticos diferenciales se deben considerar otros errores innatos del metabolismo, que también 
inician con sintomatología en el periodo neonatal y presentan hiperamonemia. Es importante recordar que si la hiperamonemia se instaura en el primer día de vida, puede tratarse de hiperamonemia transitoria, en especial si es un prematuro. La hiperamonemia puede ser secundaria a deficiencia de piruvato deshidrogenasa o aciduria glutárica tipo II, principalmente en recién nacidos a término. Si los síntomas y la hiperamonemia inician después de las 24 horas de vida, como ocurrió en nuestro paciente, la presencia de acidosis metabólica debe orientar el diagnóstico hacia $\mathrm{AO}$, deficiencia de piruvato carboxilasa, trastorno de la betaoxidación o acidosis láctica. ${ }^{11} \mathrm{En}$ un estudio de 21 pacientes con IVA que habían tenido 69 episodios de descompensaciones metabólicas, el $20 \%$ presentó hiperamonemia. ${ }^{7}$ Otros hallazgos de laboratorio incluyen hipoglucemia o hiperglucemia (esto último ocurrió en el paciente que describimos), cetonuria, hipercalcemia, anemia, trombocitopenia y leucopenia. ${ }^{12}$ En nuestro paciente, en conjunto al cuadro clínico, los resultados de amonio elevado con la acidosis metabólica con anión GAP aumentado y bicarbonato bajo orientaron el enfoque diagnóstico hacia $\mathrm{AO}$, puesto que son hallazgos comunes en este tipo de error innato en el metabolismo en el periodo neonatal. ${ }^{5}$

El análisis de ácidos orgánicos en orina se considera la herramienta de elección para la confirmación bioquímica de las AO. Más de 100 ácidos orgánicos son excretados en la orina en estas patologías y cada uno de ellos presenta un patrón excretorio característico, el cual permite llegar al diagnóstico bioquímico (Tabla 1). ${ }^{12}$ La cromatografía de ácidos orgánicos en orina de nuestro paciente mostró un perfil compatible con IVA por excreción elevada de isovalerilglicina, 3-hidroxiisovalérico, ácido isovalerilglutámico y 2-hidroxiisovalérico. Si se solicitan acilcarnitinas es posible encontrar elevación de isovalerilcarnitina (C5), la cual no es específica de IVA, ya que puede encontrarse en otros errores innatos como la aciduria metilbutírica, o también por el uso de cremas a base de ácido piválico.

Adicionalmente, se encuentra disponible el análisis molecular de la IVA. A la fecha se han descrito cerca de 40 mutaciones asociadas con IVA, siendo la mutación c.932C $>$ T (p.A282V) la más frecuente, ya que se observa en $47-52 \%$ de los pacientes detectados por el tamiz neonatal con un perfil bioquímico leve, lo anterior contrasta con las mutaciones heterogéneas descritas en pacientes con perfiles bioquímicos clásicos. ${ }^{10}$

El tratamiento para IVA puede ser más efectivo cuando el diagnóstico se realiza más tempranamente. La base son las dietas restrictivas de leucina y fórmulas basadas en aminoácidos libres de leucina, con lo cual se disminuye la producción de metabolitos tóxicos. Se debe recordar que el manejo nutricional debe proveer la energía, el nitrógeno, las vitaminas y los minerales necesarios para promover el anabolismo y crecimiento. ${ }^{7}$ Las fórmulas sintéticas deben aportar aproximadamente $50 \%$ de los requerimientos proteicos diarios.

\section{Tabla 1: Perfiles urinarios típicos de las acidemias orgánicas clásicas.}

$\begin{array}{lll}\text { Trastorno } & \text { Deficiencia enzimática } & \text { Perfil de ácidos orgánicos }\end{array}$

Acidemia isovalérica

Acidemia propiónica

Acidemia metilmalónica
Isovaleril-CoA deshidrogenasa

Propionil-CoA carboxilasa

Metilmalonil-CoA mutasa

Trastornos del metabolismo de la cobalamina
Ácido 3-hidroxi-isovalérico

Ácido 4-hidroxi-isovalérico

Isovalerilglicina

Isovalerilglutamato

Ácido 3-hidroxipropiónico

Ácido metilcítrico

Propionilglicina

Ácido 2-metilglutacónico

Ácido 3-ceto-2-metilvalérico

Ácido 3-ceto-2-metilbutírico

Tiglilglicina

Ácido 3-hidroxipropiónico

Ácido metilmalónico

Ácido metilcítrico

Propionilglicina 
La deficiencia de carnitina es frecuente, por lo que se debe realizar seguimiento periódico de los niveles de carnitina libre. El manejo adyuvante con L-carnitina y L-glicina aumenta el número de metabolitos conjugados, menos tóxicos en comparación con los metabolitos libres del ácido isovalérico. ${ }^{7,13}$

El tratamiento agudo, cuando hay descompensación metabólica, incluye el manejo de la acidosis e hiperamonemia, corrección de líquidos, glucemia y electrolitos; además de procurar la adecuada perfusión y disponibilidad de oxígeno. En el caso que se presenta, el manejo con ácido carbaglúmico tuvo un rol importante para lograr estabilizar los niveles de amonio en menos de 24 horas. El seguimiento interdisciplinario debe ser individualizado, según la edad del paciente y la severidad metabólica y clínica. Debe haber evaluación periódica de gasometría, amonio y aminoácidos cuantitativos en plasma prestando mayor atención a los niveles de isoleucina, metionina, treonina y valina, cuyos niveles pueden caer abruptamente al iniciar el tratamiento, poniendo al paciente en riesgo de desarrollar manifestaciones asociadas a la depleción de éstos, de ahí que es esencial la vigilancia nutricional. ${ }^{14}$ Por último, la asesoría genética resulta relevante, en especial para la detección prenatal en caso de que se desee un nuevo embarazo. ${ }^{15}$

\section{REFERENCIAS}

1. Vaidyanathan K, Narayanan MP, Vasudevan DM. Organic acidurias: an updated review. Indian J Clin Biochem. 2011; 26(4): 319-325.

2. Rice GM, Steiner RD. Inborn errors of metabolism (metabolic disorders). Pediatr Rev. 2016; 37(1): 3-17.
3. Ensenauer R, Fingerhut R, Maier E, Polanetz R et al. Newborn screening for isovaleric acidemia using tandem mass spectrometry: data from 1.6 million newborns. Clin Chem. 2011; 57(4): 623-626.

4. Schillaci LP, DeBrosse SD et al. Inborn errors of metabolism with acidosis organic acidemias and defects of pyruvate and ketone body metabolism. Pediatr Clin North Am. 2011; 65(2): 209-230.

5. Guglielmo RD, Giovanna G, Emanuela S, Francesco $S$ et al. "Classical organic acidurias": diagnosis and pathogenesis. Clin Exp Med. 2016; 17(3): 305-323.

6. Moorthie S, Cameron L, Sagoo GS, Bonham JR et al. Systematic review and meta-analysis to estimate the birth prevalence of five inherited metabolic diseases. J Inherit Metab Dis. 2014; 37: 889-898.

7. Daisuke F, Kimitoshi N, Hiroshi Mi, Clinical features and management of organic acidemias in Japan. J Hum Genet. 2013; 58: 769-774.

8. Ogier de Baulny H, Dionisi-Vici C, Wendel U. Branched-chain organic acidurias/acidemias. En: Saudubray JM, van den Berghe G, Walter J, editores. Inborn metabolic diseases. Berlín: Springer; 2012. pp. 277-293.

9. Vockley J, Ensenauer R, Isovaleric acidemia: New aspects of genetic and phenotypic heterogeneity. Am J Med Genet Part C. 2006; 142C: 95-103.

10. Dercksen M, Duran M, ljlst L, Mienie LJ. Clinical variability of isovaleric acidemia in a genetically homogeneous population. $\mathrm{J}$ Inherit Metab Dis. 2012; 35(6): 1021-1029.

11. Cho JM, Lee BH, Kim GH, Kim Y, et al. Chronic intermittent form of isovaleric aciduria in a 2-year-old boy. Korean J Pediatr. 2013; 56: 351-354.

12. Prieto JA, Andrade F, Aldámiz-Echevarría L, Sanjurjo P. Análisis de ácidos orgánicos en orina mediante cromatografía de gasesespectrometría de masas. Química Clínica. 2007; 26(2): 66-72.

13. Merinero $B$, Pérez-Cerdá $C$. Acidemia isovalérica. Alteraciones del catabolismo de leucina y valina. En: Sanjurjo P, Baldellou A, editores. Diagnóstico y tratamiento de enfermedades metabólicas hereditarias. Madrid: Ergon; 2014. pp. 555-568.

14. Grunert SC, Wendel $U$, Lindner $M$, Leichsenring $M$ et al. Clinical and neurocognitive outcome in symptomatic isovaleric acidemia. Orphanet J Rare Dis. 2012; 7: 9.

15. Ozgul RK, Karaca M, Kilic M, Kucuk O et al. Phenotypic and genotypic spectrum of Turkish patients with isovaleric academia. Eur J Med Genet. 2014; 57: 596-601. 